\title{
Long-term clinical results of per-oral endoscopic myotomy (POEM) for achalasia: First report of more than 10-year patient experience as assessed with a questionnaire-based survey
}

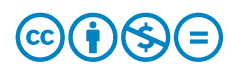

\author{
Authors \\ Manabu Onimaru, Haruhiro Inoue, Yusuke Fujiyoshi, Mary Raina Angeli Abad, Yohei Nishikawa, Akiko Toshimori, \\ Yuto Shimamura, Mayo Tanabe, Kazuya Sumi, Haruo Ikeda
}

Institution

Digestive Diseases Center, Showa University Koto Toyosu Hospital, Tokyo, Japan

submitted 24.9.2020

accepted after revision $\quad 2.11 .2020$

Bibliography

Endoscopy International Open 2021; 09: E409-E416

DOI 10.1055/a-1333-1883

ISSN 2364-3722

(c) 2021. The Author(s).

This is an open access article published by Thieme under the terms of the Creative Commons Attribution-NonDerivative-NonCommercial License, permitting copying and reproduction so long as the original work is given appropriate credit. Contents may not be used for commecial purposes, or adapted, remixed, transformed or built upon. (https://creativecommons.org/licenses/by-nc-nd/4.0/)

Georg Thieme Verlag KG, Rüdigerstraße 14,

70469 Stuttgart, Germany

Corresponding author

Manabu Onimaru, MD, PhD, Digestive Diseases Center, Showa University Koto Toyosu Hospital, 5-1-38 Toyosu, Koto-ku, Tokyo 135-8577, Japan

Fax: +81-3-6204-6396

mnbonmr0827@gmail.com

\section{ABSTRACT}

Background and study aims Since per-oral endoscopic myotomy (POEM) was introduced in 2010, it has become accepted as one of the standard treatments for esophageal achalasia worldwide. This study aimed to present long-term clinical results of POEM over 10 years and evaluate the technique and outcomes at the institution where it was first used in clinical settings.

Patients and methods Questionnaire-based surveys were sent to patients who received POEM in our institution from September 2008 to May 2010. Patient demographics and procedural outcomes and open-ended questions were posed about the postoperative courses, including symptom improvement and recurrence, additional treatments, and post-POEM gastroesophageal reflux disease (GERD) symptoms. Achalasia symptoms and post-POEM GERD symptoms were evaluated with Eckhardt scores and GerdQ systems, respectively.

Results Thirty-six consecutive POEMs were performed in that period and 10-year follow-up data were obtained from 15 patients (41.7\%). Although four cases (26.7\%) required additional pneumatic balloon dilatation (PBD), reduction in post-Eckardt scores were observed in 14 cases (93.3\%). GerdQ score was positive in one patient (6.7\%). Proton pump inhibitors (PPI) were taken by four patients (26.7\%) and their symptoms were well-controlled.

Conclusions Clinical results of POEM over 10 years were favorable regardless of various factors. Symptoms improved even in patients who required additional treatments, suggesting that POEM plays a significant role in treatment of achalasia.

\section{Introduction}

Ten years after the first report of per-oral endoscopic myotomy (POEM) for esophageal achalasia by Inoue et al [1], POEM has been established as one of the standard treatments of esophageal achalasia worldwide [2,3]. While evidence-based studies have proven POEM to be a safe and effective technique, [4,5], concerns regarding gastroesophageal reflux (GERD) after
POEM have emerged in recent years [6,7]. Along with this, the advantages and disadvantages of POEM still remain controversial; however, through an increasing number of reports and discussions, these have gradually become clearer in recent years. In addition, how to perform POEM more effectively and safely has been widely debated, and various evolutions of the indications and of techniques have been evaluated $[8,9]$. 
Bearing in mind that available literature on POEM has been limited to short- or mid-term results of up to approximately 5 years $[10,11]$, assessing the long-term clinical results of POEM in the initial period since its introduction into clinical settings was deemed to be important.

In this study, as the earliest institution to develop POEM and the largest referral center for POEM in Japan, we present our long-term clinical results of POEM over 10 years. The initial technique and outcomes are also examined and discussed.

\section{Patients and methods}

\section{Study design and patients}

This was a single-center retrospective cohort study. A database of information from patients who received POEM at Showa University from September 2008 to May 2010 was reviewed using electronic charts. Clinical surveys of patient symptoms over 10 years were conducted via phone calls or mail questionnaires. There were no exclusion criteria in this follow-up study; however, patients under 18 years of age or those with advanced sigmoid type achalasia were excluded from the indications for POEM procedure over the study period.

\section{Procedural technique of POEM}

The POEM procedure was performed as previously described in our first report of 17 consecutive POEM patients [1]. Of them, seven cases (Cases 1-7) were also included in this study. The procedure was done with the patient under general anesthesia with positive pressure ventilation. A submucosal tunnel was created on the posterior (5 o'clock axis) or anterior (2 o'clock axis) side from the level of the mid- or lower esophagus downwards about 1 to $3 \mathrm{~cm}$ into the proximal stomach passing the esophagogastric junction (EGJ). In all cases, myotomy was also carried out with selective circular muscle cutting. An adequate myotomy on the gastric side was confirmed by the endoscopic appearance, such as the insertion length of the endoscopy, a prompt decrease in resistance when entering into the stomach side passing through EG], and recognition of the palisade vessels in the esophagus and submucosal spindle vessels in the stomach. Confirmation by double-scope [8] or other methods [9] was not performed at that time. All procedures were performed by one expert endoscopist who was the first in the world to pioneer POEM.

\section{Follow-up measurements}

The surveys conducted during this study consisted of openended questions regarding the postoperative course, such as symptom improvement and recurrence, additional treatment, and GERD symptoms after POEM. Achalasia symptoms and post-POEM GERD symptoms were evaluated with Eckardt score and GerdQ systems [12], respectively. Esophagogastroduodenoscopy (EGD), barium swallow, and 24-hour impedance-pH monitoring (MII-pH) were added in Case 11 (32 ${ }^{\text {nd }}$ experience with POEM for our team) because the patient answered that he had both insufficient results from POEM and a positive GerdQ score after POEM.

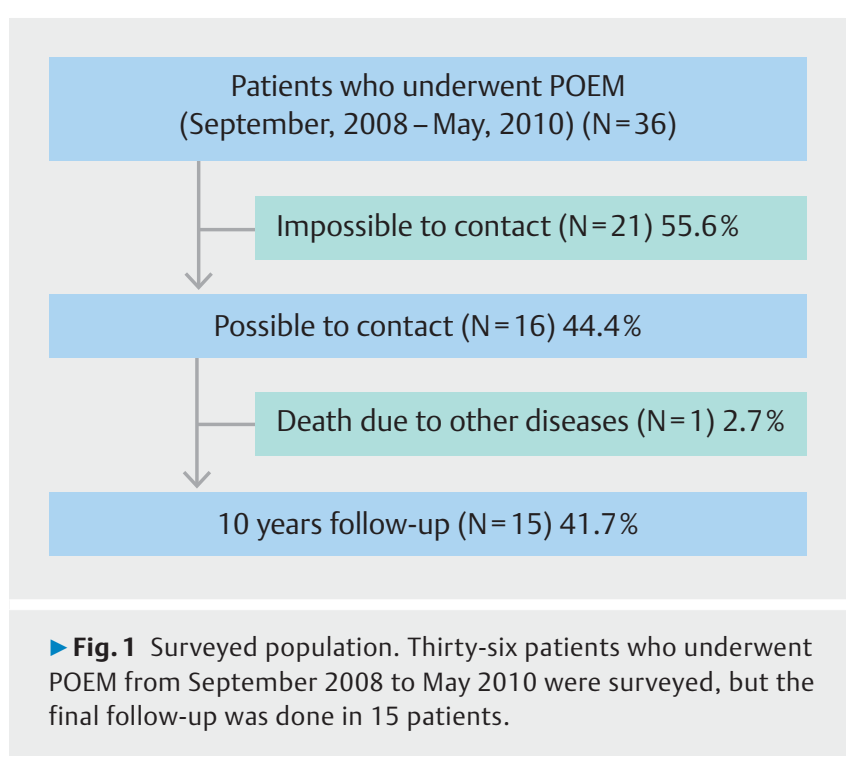

\section{Definition of outcome measurements}

The degree of esophageal dilatation and type were classified by barium esophagogram. According criteria from the Japan Society of Esophageal Diseases, the degree of esophageal dilatation was classified by the maximum diameter of the esophageal lumen into grade I $(<3.5 \mathrm{~cm})$, grade II $(<3.5-6.0 \mathrm{~cm})$, and grade III $(>6 \mathrm{~cm})$, and the type of achalasia was classified by the shape of the esophageal lumen as straight (St), sigmoid (Sg), and advanced sigmoid (aSg) [13].

The primary endpoint of this study was symptom improvement by obtaining a reduction in Eckardt score at 10 years after POEM. The secondary endpoint was GERD symptoms at 10 years after POEM. Positive GERD symptoms were defined as a post-POEM GerdQ score of 8 or more according to the previous report [12].

\section{Ethical considerations}

This study was approved by the Ethics Committee of Showa University Koto Toyosu Hospital (IRB Registration No:20T7022). Written informed consent was obtained from all participants.

\section{Statistical analysis}

Median (minimum-maximum range) was used to report continuous and categorical variables. All analyses were performed using JMP Pro 14.0.0 (SAS Institute Inc., North Carolina, United States).

\section{Results}

Between September 2008 and May 2010, POEM was performed on 36 consecutive patients in our institution. We were able to follow up with 16 of them (44.4\%) via phone calls or mail questionnaires. However, one of the 16 patients died from another disease 2 years after POEM. Therefore, 10 years of follow-up data from a total of 15 patients (41.7\%) were analyzed in this study ( Fig. 1). 
II

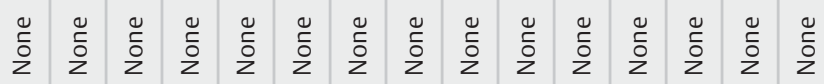

III!

Mis

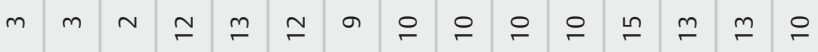

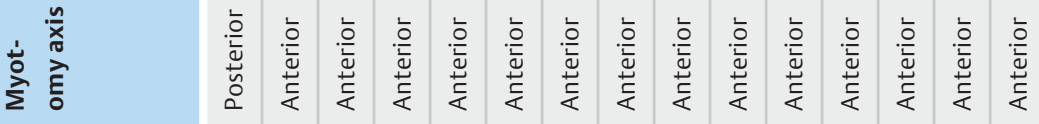

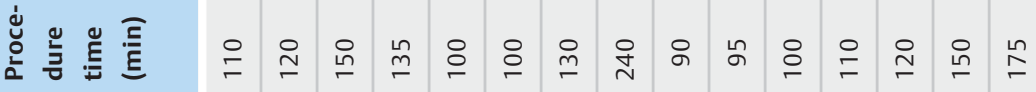

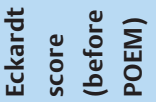

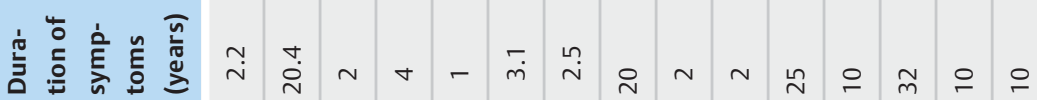

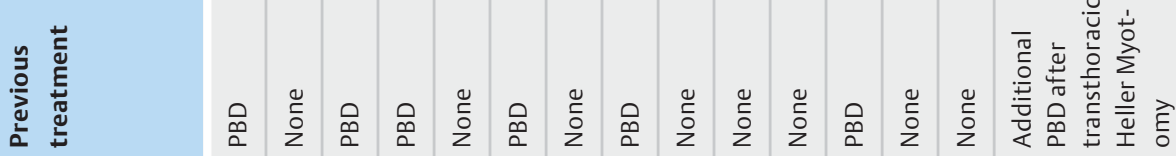

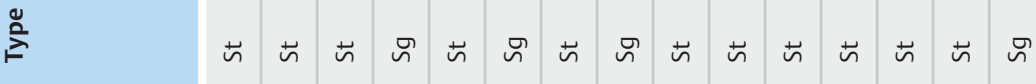

:= = = = =

ভ

这

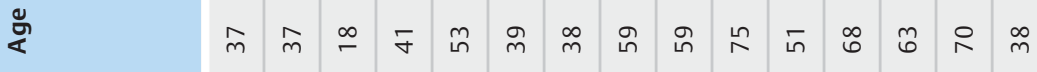

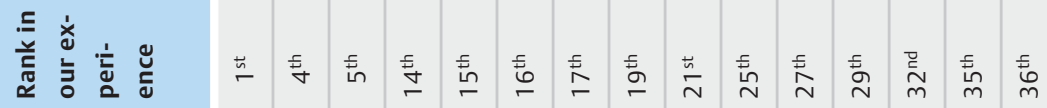

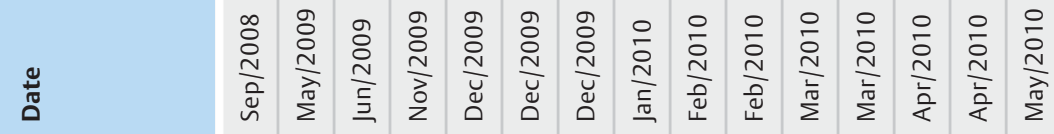

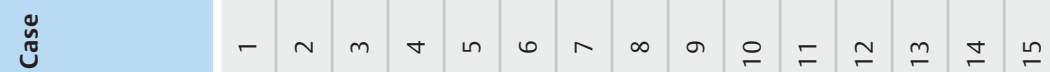




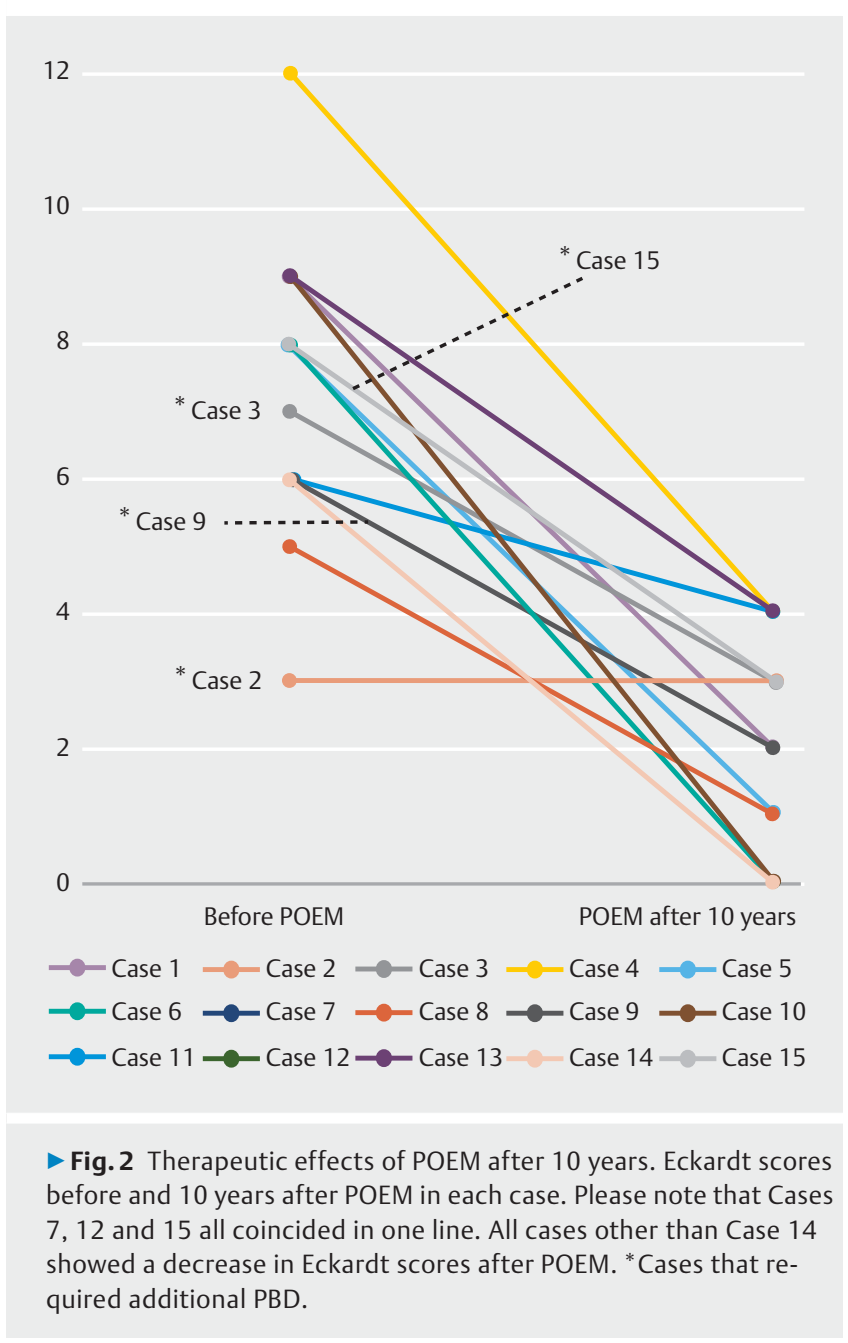

Patient demographics and procedural data are shown in - Table 1. In the initial period, POEM was indicated for all typical achalasia except in patients under 18 years old or who had advanced sigmoid type achalasia. The median age was 51 years (range 18-75) and the median duration of symptoms was 4 years (range $1-32$ ). Seven patients (46.7\%) were male, three (20.0\%) had grade III dilation, and four (26.7\%) had sigmoid type achalasia. Seven patients $(46.7 \%)$ received previous treatment with pneumatic balloon dilatation (PBD). In one case (Case 15), previous PBD was done as an additional treatment after transthoracic Heller myotomy because of its insufficient effect. The median pre-POEM Eckardt score was 8 (range 3-12).

In the first case (Case 1), myotomy on the posterior side was selected, but in all the latter cases (Cases 2-15), myotomy was switched to the anterior side (2 o'clock). This is due to the presence of the spine behind the esophagus, which destabilized the appropriate motion of the endoscopic tips. In addition, anterior myotomy can avoid destroying the angle of His in the light of preventing the risk of post-POEM GERD. Furthermore, because the procedure at that time was focused on opening the EGJ and consensus about the appropriate length of myotomy had not yet been reached, the length of myotomy in the esophagus side varied from 2 to $15 \mathrm{~cm}$, and a minimum of $1 \mathrm{~cm}$ on the stomach side. Based on our experience with the first seven cases (Cases 1, 2, and 3 in this paper), the myotomy length on the esophageal side was relatively short. After subsequent reports that a $7-\mathrm{cm}$ myotomy was generally recommended to achieve complete release of LES in surgical Heller myotomy, myotomy on the esophageal side was extended to over $8 \mathrm{~cm}$ in the latter cases [14]. The median procedural time was 120 minutes (range 90-240) and no adverse events occurred in this series.

Symptomatic results in each case are shown in $>$ Fig. 2 and - Table 2. As shown in > Fig. 2, although four patients (Cases $2,3,9$, and 15 ) required additional treatments (shown with asterisk in > Fig. 2), symptom improvement was obtained in 14 of 15 patients $(93.3 \%)$. Table 2 shows the details of the clinical course in each case. Cases 4, 11, and 13 had a post-Eckardt score of 4 , which was generally considered inadequate relief of achalasia symptoms. In case 13 , the patient had a temporary feeling of symptom recurrence 1 month after POEM, but in Cases 4 and 13, they were satisfied with the results at present without further treatment. In Case 11, the patient was satisfied and achalasia symptom improvement was noted, but an uncomfortable feeling of GERD symptoms was reported. Additional PBD was required in Cases 2, 3, 9, and 15. The duration of the recurrence of symptoms was 1 to 5 years after POEM. Additional PBD was effective in Cases 2, 3, and 9, with relief of symptoms to some extent. Meanwhile, symptom recurrence at 5 years after POEM was noted in Case 15; however, due to the mildness of the symptoms, the patient did not seek any treatment. The patient's symptoms eventually worsened, prompting him to finally seek care and he subsequently received an additional PBD 9 years after POEM. Despite the additional PBD, no symptom improvement was noted, yet the patient was able to tolerate the symptoms afterwards.

Post-POEM GERD data are shown in > Table 2. Four of 15 patients $(26.7 \%)$ are taking proton pump inhibitors (PPIs). Of them, two patients (Cases 2 and 4) are taking PPIs at their request to prevent GERD symptoms despite being asymptomatic. The patient in Case 8 only takes PPIs when symptoms occur, whereas the patient in Case 12 takes PPis daily. In both patients, the symptoms are well controlled.

Meanwhile, one patient (Case 11) (6.7\%) had a positive GerdQ score without any PPI intake. As such, repeat evaluation was performed, which included EGD, barium swallow, and MII$\mathrm{pH}$. EGD findings revealed inadequate opening of the EGJ and large diverticulum just above the EG]. Barium swallow also revealed retention of barium in the esophagus with a large diverticulum above the EGJ ( $\triangleright$ Fig. 3). MII-pH showed that percent time clearance $\mathrm{pH}$ was $0.0 \%$ and DeMeester composite score was 0.9 , which indicated no GERD in this patient.

\section{Discussion}

In the present paper, the experience at the earliest institution to develop POEM and the largest referral center for the technique in Japan, including 10-year clinical results based on postPOEM GERD data, is presented. POEM has been reported to be a highly effective, minimally invasive treatment for achalasia and 


\begin{tabular}{|c|c|c|c|c|c|c|c|c|c|c|c|c|c|c|c|}
\hline$\overline{\frac{a}{2}}$ & 1 & $\begin{array}{l}\frac{5}{3} \\
\text { 齐 } \\
\frac{5}{5}\end{array}$ & 1 & $\begin{array}{l}\frac{5}{3} \\
\frac{5}{5} \\
\frac{5}{5}\end{array}$ & 1 & 1 & 1 & 总 & 1 & 1 & 1 & 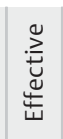 & 1 & 1 & 1 \\
\hline 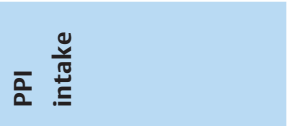 & $\stackrel{\circ}{z}$ & $\stackrel{\tilde{x}}{\succ}$ & ì & 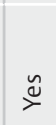 & i & $\stackrel{0}{z}$ & $\stackrel{0}{z}$ & 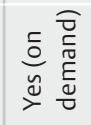 & z & z & $\stackrel{0}{z}$ & $\stackrel{\check{\nu}}{\check{\nu}}$ & $\stackrel{0}{z}$ & $\stackrel{0}{z}$ & $\stackrel{\circ}{z}$ \\
\hline 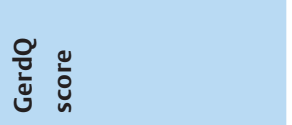 & 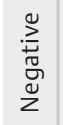 & 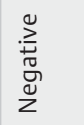 & 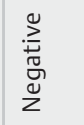 & 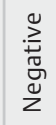 & 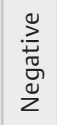 & 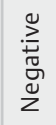 & $\begin{array}{l}\sum_{\stackrel{D}{0}}^{0} \\
\stackrel{\Xi}{Z}\end{array}$ & 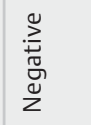 & 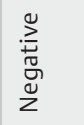 & 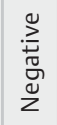 & $\sum_{\substack{\bar{n} \\
\vdots}}^{0}$ & 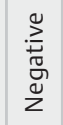 & 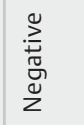 & 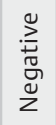 & 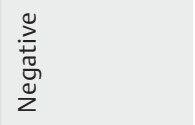 \\
\hline 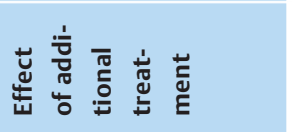 & & 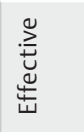 & 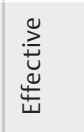 & 1 & & & & & 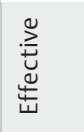 & & 1 & & 1 & & 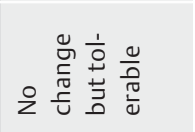 \\
\hline 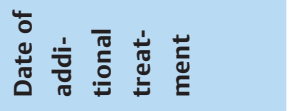 & & 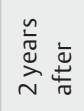 & 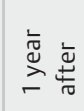 & , & & & & & 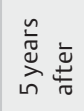 & & 1 & & 1 & & 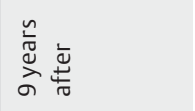 \\
\hline 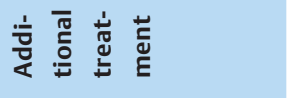 & & 命 & 合 & $\begin{array}{l}\text { Ĭ } \\
\text { ż }\end{array}$ & & & & & 命 & & $\begin{array}{l}\stackrel{0}{0} \\
\text { ż }\end{array}$ & & 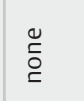 & & 合 \\
\hline 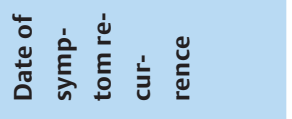 & & 峁 & 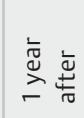 & 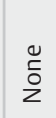 & & & & & 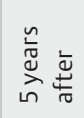 & & 气̆ & & 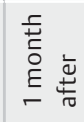 & & 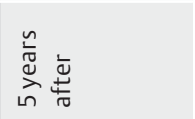 \\
\hline 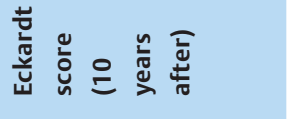 & $\sim$ & $m$ & $m$ & $\sigma$ & - & 0 & $m$ & - & $\sim$ & 0 & $\nabla$ & $m$ & $\nabla$ & 0 & $m$ \\
\hline 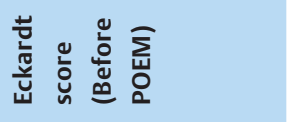 & $a$ & $m$ & $r$ & $\stackrel{\sim}{\sim}$ & $\infty$ & $\infty$ & $\infty$ & in & 0 & $\sigma$ & 0 & $\infty$ & $a$ & 0 & $\infty$ \\
\hline 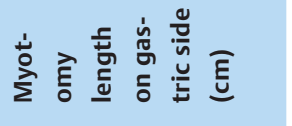 & $\sim$ & - & - & $N$ & - & $m$ & $m$ & $m$ & $m$ & $m$ & $m$ & $m$ & - & $N$ & $\sim$ \\
\hline 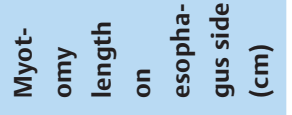 & $m$ & $m$ & $\sim$ & $\simeq$ & $\stackrel{m}{r}$ & $\simeq$ & $a$ & 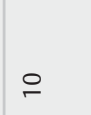 & $\stackrel{\circ}{\circ}$ & $\stackrel{\circ}{\circ}$ & $\stackrel{\circ}{\circ}$ & $\stackrel{\llcorner}{\sim}$ & $\stackrel{m}{r}$ & $\stackrel{m}{r}$ & $\stackrel{\circ}{-}$ \\
\hline 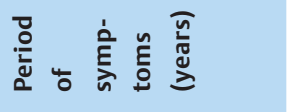 & $\stackrel{\sim}{\sim}$ & ¿े. & $\sim$ & $\nabla$ & - & $\bar{m}$ & $\stackrel{\stackrel{L}{\sim}}{\sim}$ & $\stackrel{\sim}{\sim}$ & $N$ & $\sim$ & $\stackrel{\llcorner}{\sim}$ & $\stackrel{\circ}{-}$ & m & $\stackrel{\circ}{-}$ & $\stackrel{\circ}{\circ}$ \\
\hline 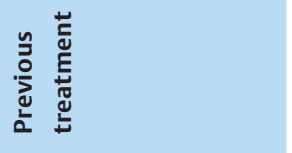 & $\stackrel{\otimes}{\varrho}$ & $\frac{\tilde{0}}{2}$ & 合 & 啇 & $\frac{\mathscr{0}}{0}$ & @ि & 芩 & ळि & 㫕 & 气̊ & 气̊ & $\stackrel{0}{\circ}$ & 号 & 总 & 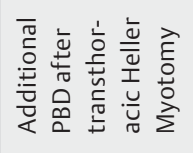 \\
\hline 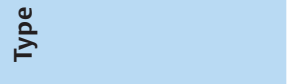 & $\ddot{n}$ & 㫕 & $\ddot{n}$ & $\Xi$ & $\hbar$ & $\varpi$ & $\dot{\omega}$ & $\Xi$ & $\ddot{n}$ & $\ddot{\omega}$ & $\ddot{n}$ & 㟧 & 虫 & $\ddot{\omega}$ & $\Xi$ \\
\hline 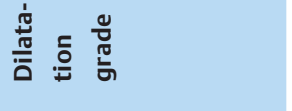 & $=$ & $=$ & $=$ & $\equiv$ & $=$ & $\equiv$ & - & $\equiv$ & - & $=$ & - & - & $=$ & - & $=$ \\
\hline ư & - & $\sim$ & $m$ & $\nabla$ & in & 0 & $r$ & $\infty$ & $a$ & $\stackrel{\circ}{\circ}$ & $=$ & $\simeq$ & $\stackrel{m}{r}$ & 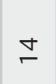 & $\stackrel{\llcorner}{\leftarrow}$ \\
\hline
\end{tabular}




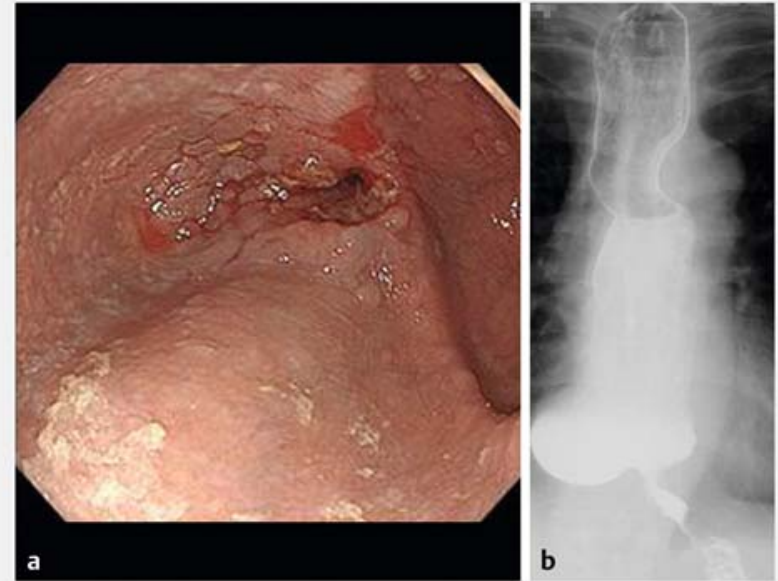

- Fig. 3 Findings from EGD and barium swallow in Case 11. a EGD showed inadequate opening of the EG], mild erosive GERD, and a large diverticulum just above the EGJ. b Barium swallow also revealed retention of barium in the esophagus with a large diverticulum above the EGJ.

related esophageal motility disorders with short-term followup $[4,15]$; however, to our knowledge, this is the longest follow-up report and the first report of over 10 years of clinical results with POEM.

Since the introduction of POEM into clinical practice based on the evidence of an experimental report on the safety of endoscopic myotomy in a porcine model by Pasricha et al [16], indications initially were restricted for only typical achalasia; hence, POEM has been safely completed in all cases regardless of prior treatment. Based on primary reports regarding efficiency and safety associated with short-term results of POEM, indications for the procedure have been expanded $[15,17$ 19], and to date, modifications and discussions are still being done among endoscopists all over the world to achieve safer and better results $[20,21]$.

The majority of patients in this study obtained symptom improvement 10 years after POEM, suggesting that the clinical efficacy of POEM is favorable. On the other hand, four patients (Cases 2, 3, 9, and 15) required additional PBD treatment during their clinical courses. Based on previous literature, patient demographics factors associated with poor results of POEM were reported to be male gender, high pre-Eckardt score, longer duration of achalasia symptoms over 10 years, prior treatments, dilated esophagus, sigmoid type achalasia, and type III Chicago classification [22-25]. However, in the present study, tendencies toward these factors were not elucidated due to the small sample size. In addition, procedural factors such as myotomy length also were reported to have an effect on POEM results. According to clinical practice guidelines for POEM in Japan [2], to secure a complete LES incision, 1- to 2-cm myotomy into the gastric side is recommended. In our series, in four cases (Case 2, 3, 5 and 13), a shorter 1 -cm myotomy was made in the stomach. In two of these patients (Case 2 and 3), additional PBD was required and one patient (Case 13) had a feeling of symptom recurrence 1 month after POEM and had a postPOEM Eckardt score of 4. Our study could not draw concrete conclusions about the importance of the length of myotomy on the gastric side, but taking into account previous reports, we cannot totally rule out that these results may have been influenced by the shorter gastric myotomy length. Another interesting result was that in most of the cases with symptom recurrence, the recurrence occurred a short time after POEM, and additional PBD resulted in improvement in symptoms. This suggests that even if the myotomy length is insufficient, it is possible that this previous myotomy site can become the "starting point" for the dilatation since it has already been cut, hence, enhancing the effects of the additional PBD. To avoid compromising the effects of POEM due to insufficient myotomy length, a double-scope method has been used routinely in recent years in our center to intraoperatively confirm adequacy of myotomy length on the gastric side [8]. In Case 15, POEM was done as a "second myotomy" following the insufficient effect of the first transthoracic surgical Heller myotomy and PBD. Symptom improvement to some extent was noted. Although a slight exacerbation occurred after 5 years, symptoms were still tolerable for the patient. Nine years after POEM, additional PBD was carried out to improve her symptoms; however, it did not provide relief. Since this case had several factors such as sigmoid type, long duration of symptoms, and prior treatment, complete relief of symptoms might not be obtained only by making an opening in the LES. Still, POEM was considered to have a role in alleviating this patient's symptoms to some extent.

Despite the high efficacy and safety rates for POEM, the onset of GERD after POEM has become a concern and it is now an important issue for discussion. Apart from Heller myotomy with antireflux surgery, adjacent structures surrounding the distal esophagus, which work as one of the major natural barriers to reflux, are potentially preserved in POEM; however, post-POEM GERD has been reported, ranging from $14 \%$ to $57 \%[5,7,26$, 27]. In our cases, four patients (Cases 2, 4, 8, and 12) were prescribed PPIs. Of these, two patients (Cases 2 and 4 ) took PPIs for prophylaxis regardless of being asymptomatic, and in the other two patients (Cases 8 and 12), symptoms were controlled well with PPIs. Based on available reports, factors that may increase the risk of post-POEM GERD include the following: a myotomy length on the gastric side $>2.5 \mathrm{~cm}$, female sex, low pre-POEM LES pressure, full-thickness myotomy, and posterior myotomy [6, 27-30]. However, the present study did not reveal a tendency toward these factors affecting GERD symptoms. Only Case 11 had a positive GerdQ score. In this case, although the length of myotomy in the stomach was $3 \mathrm{~cm}$, the therapeutic effect was not sufficient, and the patient still exhibited a post-POEM Eckardt symptom score of 4 . Considering the emergence of a diverticulum just above the EG] after POEM and a poor outflow of barium in the esophagogram, the positive GerdQ score might not represent acid reflux from the stomach caused by post-POEM GERD, but instead, reflux of esophageal residue due to an insufficient therapeutic effect of POEM. This consideration was also supported by the results of MII-pH. We believe that additional treatment, such as PBD or a second POEM, is required in such cases after detailed examinations, including 
high-resolution manometry (HRM) and the indications must be carefully considered.

Certain limitations of this study must be acknowledged. Aside from its single-center retrospective nature, the sample size was relatively small. Contacting patients more than 10 years after treatment posed challenges, such as ascertaining their current whereabouts, which is why we were able to reach only $44.4 \%$ of them. Moreover, there is a lack of objective analysis. Our hospital was the only facility performing POEM 10 years ago, and most of the patients at that time came from far away to receive POEM. Because most of them were satisfied with their past results with POEM, unfortunately, we could not get consent from them to come all the way to our facility just for the follow-up examinations. Furthermore, the COVID-19 pandemic made it difficult to perform follow-up and provide usual examinations. Finally, POEM procedures in this series had not yet been standardized 10 years ago, and methods to stabilize the therapeutic effect and safety were not yet established. This consequently resulted in various biases in considering the clinical outcomes in this study. Therefore, a multicenter study with objective data is needed in the near future.

These are the longest-term clinical results with POEM to date. The clinical results of POEM were satisfying enough regardless of various patient and procedural factors. Even in cases for which additional treatment was required, symptoms scores improved to below their pre-POEM baselines, suggesting that the technique plays a significant role in treatment of achalasia.

\section{Conclusion}

In conclusion, this study showed satisfactory long-term clinical results with POEM over 10 years. We believe this study provides important information and lessons that can be applied to future POEM treatment.

\section{Acknowledgements}

The authors thank all the patients and the clinical staff for their participation and contribution to this study.

\section{Competing interests}

Dr. Inoue is an advisor for Olympus Corporation and Top Corporation. He has also received educational grants from Olympus Corp. and Takeda Pharmaceutical Co.

\section{References}

[1] Inoue H, Minami H, Kobayashi Y et al. Peroral endoscopic myotomy (POEM) for esophageal achalasia. Endoscopy 2010; 42: 265-271

[2] Inoue H, Shiwaku H, Iwakiri K et al. Clinical practice guidelines for peroral endoscopic myotomy. Dig Endosc 2018; 30: 563-579

[3] Zaninotto G, Bennett C, Boeckxstaens G et al. The 2018 ISDE achalasia guidelines. Dis Esophagus 2018; 31: 1-29

[4] Kahrilas PJ, Katzka D, Richter JE. Clinical Practice Update: The Use of Per-Oral Endoscopic Myotomy in Achalasia: Expert Review and Best
Practice Advice From the AGA Institute. Gastroenterology 2017; 153 : $1205-1211$

[5] Shiwaku H, Inoue H, Sato H et al. Peroral endoscopic myotomy for achalasia: a prospective multicenter study in Japan. Gastrointest Endosc 2020; 91: 1037-1044, e1032

[6] Inoue H, Shiwaku H, Kobayashi Y et al. Statement for gastroesophageal reflux disease after peroral endoscopic myotomy from an international multicenter experience. Esophagus 2020; 17: 3-10

[7] Repici A, Fuccio L, Maselli R et al. GERD after per-oral endoscopic myotomy as compared with Heller's myotomy with fundoplication: a systematic review with meta-analysis. Gastrointest Endosc 2018; 87 : 934-943, e918

[8] Grimes KL, Inoue H, Onimaru M et al. Double-scope per oral endoscopic myotomy (POEM): a prospective randomized controlled trial. Surg Endosc 2016; 30: 1344-1351

[9] Rieder E, Swanstrom LL, Perretta S et al. Intraoperative assessment of esophagogastric junction distensibility during per oral endoscopic myotomy (POEM) for esophageal motility disorders. Surg Endosc 2013; 27: 400-405

[10] Li QL, Wu QN, Zhang XC et al. Outcomes of per-oral endoscopic myotomy for treatment of esophageal achalasia with a median followup of 49 months. Gastrointest Endosc 2018; 87: 1405-1412, e1403

[11] Teitelbaum EN, Dunst CM, Reavis KM et al. Clinical outcomes five years after POEM for treatment of primary esophageal motility disorders. Surg Endosc 2018; 32: 421-427

[12] Jones R, Junghard O, Dent J et al. Development of the GerdQ, a tool for the diagnosis and management of gastro-oesophageal reflux disease in primary care. Aliment Pharmacol Ther 2009; 30: 1030-1038

[13] Japan Esophageal Society. Descriptive Rules for Achalasia of the Esophagus, 4th Edition. Esophagus 2017; 14: 275-289

[14] Woltman TA, Pellegrini CA, Oelschlager BK. Achalasia. Surg Clin North Am 2005; 85: 483-493

[15] Chandan S, Mohan BP, Chandan OC et al. Clinical efficacy of per-oral endoscopic myotomy (POEM) for spastic esophageal disorders: a systematic review and meta-analysis. Surg Endosc 2020; 34: 707-718

[16] Pasricha PJ, Hawari R, Ahmed I et al. Submucosal endoscopic esophageal myotomy: a novel experimental approach for the treatment of achalasia. Endoscopy 2007; 39: 761-764

[17] Chone A, Familiari P, von Rahden B et al. Multicenter evaluation of clinical efficacy and safety of per-oral endoscopic myotomy in children. J Pediatr Gastroenterol Nutr 2019; 69: 523-527

[18] Sanaka MR, Chadalavada P, Alomari M et al. Peroral endoscopic myotomy is a safe and effective treatment modality for geriatric patients with achalasia. Esophagus 2020; 17: 484-491

[19] Hu JW, Li QL, Zhou PH et al. Peroral endoscopic myotomy for advanced achalasia with sigmoid-shaped esophagus: long-term outcomes from a prospective, single-center study. Surg Endosc 2015; 29: 2841-2850

[20] Nabi Z, Ramchandani M, Sayyed M et al. Comparison of short versus long esophageal myotomy in cases with idiopathic achalasia: a randomized controlled trial. J Neurogastroenterol Motil 2020: doi:10.5056/jnm20022

[21] Khashab MA, Sanaei O, Rivory J et al. Peroral endoscopic myotomy: anterior versus posterior approach: a randomized single-blinded clinical trial. Gastrointest Endosc 2020; 91: 288-297, e287

[22] Oude Nijhuis RAB, Prins LI, Mostafavi N et al. Factors associated with achalasia treatment outcomes: systematic review and meta-analysis. Clin Gastroenterol Hepatol 2020; 18: 1442-1453

[23] Ren Y, Tang X, Chen Y et al. Pre-treatment Eckardt score is a simple factor for predicting one-year peroral endoscopic myotomy failure in patients with achalasia. Surg Endosc 2017; 31: 3234-3241 
[24] Liu ZQ, Li QL, Chen WF et al. The effect of prior treatment on clinical outcomes in patients with achalasia undergoing peroral endoscopic myotomy. Endoscopy 2019; 51: 307-316

[25] Kumbhari V, Tieu AH, Onimaru M et al. Peroral endoscopic myotomy (POEM) vs laparoscopic Heller myotomy (LHM) for the treatment of Type III achalasia in 75 patients: a multicenter comparative study. Endosc Int Open 2015; 3: E195-E201

[26] Nabi Z, Ramchandani M, Kotla R et al. Gastroesophageal reflux disease after peroral endoscopic myotomy is unpredictable, but responsive to proton pump inhibitor therapy: a large, single-center study. Endoscopy 2020; 52: 643-651

[27] Kumbhari V, Familiari P, Bjerregaard NC et al. Gastroesophageal reflux after peroral endoscopic myotomy: a multicenter case-control study. Endoscopy 2017; 49: 634-642
[28] Grimes KL, Bechara R, Shimamura Y et al. Gastric myotomy length affects severity but not rate of post-procedure reflux: 3-year follow-up of a prospective randomized controlled trial of double-scope per-oral endoscopic myotomy (POEM) for esophageal achalasia. Surg Endosc 2020; 34: 2963-2968

[29] Wang XH, Tan YY, Zhu HY et al. Full-thickness myotomy is associated with higher rate of postoperative gastroesophageal reflux disease. World J Gastroenterol 2016; 22: 9419-9426

[30] Mota RCL, de Moura EGH, de Moura DTH et al. Risk factors for gastroesophageal reflux after POEM for achalasia: a systematic review and meta-analysis. Surg Endosc 2020: doi:10.1007/s00464-02007412-y 